\title{
Overview of non-steroidal anti-inflammatory drugs (nsaids) in resource limited countries
}

\begin{abstract}
The non-steroidal anti-inflammatory drugs (NSAIDs) is a drug class that groups together drugs that provide analgesic (pain-killing) and antipyretic (fever-reducing) effects, and, in higher doses, anti-inflammatory effects. The analgesic and anti-inflammatory properties acts by inhibiting two recognized isoenzymes of prostaglandin $\mathrm{G} / \mathrm{H}$ synthase also known as cycl-oxygenase (COX), which are COX 1 and COX 2.The pharmacodynamic action of these drugs is mostly mediated by inhibition of COX2, while the adverse reactions are largely due to COX1 inhibition.. The NSAID selectively inhibiting COX2 were developed in the 90s to reduce the risk of gastrointestinal toxicity. NSAID use has been found to be associated with an increased risk of heart failure in several randomized clinical trials and observational studies. Nevertheless, there is still limited information on the risk of heart failure associated with the use of individual NSAIDs (both COX 2 inhibitors and traditional NSAIDs) in clinical practice in resource poor communities, and especially on their doseresponse associations. The most prominent members of this group of drugs, are aspirin, ibuprofen, naproxen, that are all available over the counter in most countries. Paracetamol (acetaminophen) is generally not considered an NSAID because it has only little antiinflammatory activity. It treats pain mainly by blocking COX-2 mostly in the central nervous system, but not much in the rest of the body. Most NSAIDs inhibit the activity of (COX-1) and COX2, and thereby the synthesis of prostalglandin and thromboxanes. It is thought that inhibiting COX-2 leads to the anti-inflammatory, analgesic and antipyretic effects and that those NSAIDs also inhibiting COX-1, particularly aspirin, may cause gastrointestinal bleeding and ulcers. This review attempts to give a comprehensive view of NSAIDs pharmacological activities, the drug class, mode of action and usage in a low income economy.
\end{abstract}

Keywords: non-steroidal anti-inflammatory, drug low income economy, prostaglandin, thromboxane, low income economy
Volume 4 Issue I - 2018

\author{
Charles Ntungwen Fokunang,' Estella Tembe \\ Fokunang,' Kechia Frederick, ${ }^{2}$ Bathelemy \\ Ngameni, ${ }^{3}$ Bonaventure Ngadjui ${ }^{3}$ \\ 'Department of Pharmacotoxicology and Pharmacokinentics, \\ University of Yaoundé, Cameroon \\ ${ }^{2}$ Department of Microbiology, University of Yaoundé, Cameroon \\ ${ }^{3}$ Department of Phytochemistry and Pharmacognosy, University
} of Yaoundé, Cameroon

\section{Correspondence: Charles Fokunang, Department of} Pharmacotoxicology and Pharmacokinentics, Faculty of Medicine and Biomedical Sciences, University of Yaoundé, Cameroon, Tel +237 670902446, Email charlesfokunang@yahoo.co.uk

Received: December 19, 2017 | Published: January 09, 2018
Abbreviations: NSAIDs, non-steroidal anti-inflammatory drugs; COX, cyclo-oxygenase; GI, gastrointestinal; DDD, defined daily doses

\section{Introduction}

Non-steroidal anti-inflammatory drugs (NSAIDs) belong to a wide class of therapeutic agents with analgesic and anti-inflammatory properties that inhibit the two recognized isoenzymes of prostaglandin $\mathrm{G} / \mathrm{H}$ synthase (also known as cyclo-oxygenase (COX)) known as, COX 1 and COX $2 \cdot{ }^{1,2}$ Since the therapeutic action of these drugs is highly mediated by inhibition of COX 2, and their gastrointestinal adverse reactions are largely due to COX 1 inhibition. The NSAIDs selectively inhibiting COX 2 was developed in the 1990s to reduce the risk of gastrointestinal toxicity in the clinical settings. ${ }^{3}$ Non-steroidal anti-inflammatory drugs (NSAIDs) are the most widely prescribed drugs for the treatment of pain and inflammation in many conditions, including osteoarthritis and rheumatoid arthritis. ${ }^{3,4}$ The COX-2 selective drugs are the newer drugs that have recently been made available in the drug market, and some examples include nimesulide, nabumatone, meloxicam, etodolac, celecoxib, and rofecoxib. ${ }^{5,6}$ The last two have become popular amongst clinicians as they are generally considered to be more safe and tolerable, and at least equally efficacious with maximum therapeutic output. There are however many issues that need to be addressed before the COX-2 widespread usage can be recommended in situations where conventional NSAIDs are helpful. ${ }^{7}$
The need for newer NSAIDs has led to the understanding that the inhibition of the COX-1 isoform is responsible for the side effects of the conventional NSAIDs and the COX-2 is responsible for the beneficial effects such as the anti-inflammatory effects and analgesia. ${ }^{8,9}$ The COX-2 is an inducible isoform and its synthesis is increased at the sites of inflammation. However, there are many concerns that have been discussed regarding these drugs. There is evidence that the COX-2 is already becoming popular drugs in many countries despite of being expensive. ${ }^{10}$ Some important guidelines have been developed to support clinicians in selecting NSAIDs for usage.

\section{Importance in selecting the right NSAID in a clinical setting}

The question arises as to why it is necessary to choose a particular NSAID when all have similar pharmacological profile? The answer is that their safety, tolerability, and efficacy differ in clinical situations. Aspirin has dominated the pharmaceutical market for more than 50 years ever since its synthesis in 1899 and physicians have no other choice but to go for it. ${ }^{11,12}$ The scenario had only started changing in the early 1950s when other NSAIDs started hitting the drug stores and the question of choosing a particular drug started getting importance. NSAIDs presently are the most widely used drugs in medicine and their annual sales in the world are more than 6billion dollars. ${ }^{13}$

Presently, more than 100 NSAIDs have been tested clinically and more than 50 are there in the world market. ${ }^{13,14}$ Nearly 35 million people are taking them on daily basis and FDA has ranked them the 
most frequent cause of adverse drug reactions. ${ }^{15}$ Unfortunately, they also cause most frequently lethal drug toxicity such as gastrointestinal haemorrhage. Importantly, nonprescription use, that is often ignored, is considered to be seven folds higher than the prescription use. ${ }^{16}$ The advent of newer drugs in the market makes the question of NSAID choice all the more important as exemplified by the fact that 2.5 million prescriptions were written for celecoxib alone under 3 months of its release. ${ }^{16,17}$ The decision of using NSAIDs in most therapeutic situation is empirical, but certain principles can help clinicians prescribing them safely and effectively. These principles can be reviewed as follows;

Choosing an NSAID: Choosing an NSAID for its analgesic and antipyretic effect in indications like fever, common cold, dental pain, minor soft tissue injuries, musculo-skeletal pain, and non-specific body aches is not difficult as in most circumstances the drug is to be used for a short duration only. ${ }^{17-19}$ Both newer drugs, e.g., celecoxib and rofecoxib have now been approved by FDA for short-term relief of pain and inflammation.

Clinicians need to acquaint themselves with minimal number of drugs: for instance most rheumatologists are of the opinion that clinicians should familiarize themselves with a dozen of NSAIDs and try to get full information about them. ${ }^{7,20}$ Under most circumstances, this list should not generally exceed 20 drugs so that safe and effective use of the drugs can be achieved.

Choice of NSAID: Choice of NSAID for chronic and disabling inflammatory joint diseases like rheumatoid arthritis and osteoarthritis is governed by age, diagnosis, degree of severity, relative gastrointestinal safety, tolerability, and relative efficacy in the given clinical situation. ${ }^{21}$ It is a common misconception that all NSAIDs are equally therapeutically efficacious and any one of them could be used for the given indication. However, the use of multiple NSAIDs should be strongly discouraged as an agent with comparatively less GI side effects like ibuprofen and diclofenac should be preferred in place of indomethacin, piroxicam, or naproxan, which are more gastrotoxic. ${ }^{5,22}$ In situations, e.g., osteoarthritis where inflammation of joints is minimal, analgesics like paracetamol should be preferred over antiinflammatory drugs like ibuprofen. The American Rheumatological Association recommends the use of $1 \mathrm{gm}$ of paracetamol every 6 hours for pain relief in osteoarthritis. ${ }^{13,23}$ In situations where diagnosis is uncertain, the drug should be empirically chosen and given for a week or so and if the response is adequate it should be continued until side effects mandate its withdrawal. Ankylosing spondylitis, for unknown reasons, responds better to a particular NSAID like indomethacin. It is probably related to its stronger inhibition of prostaglandin synthesis., ${ }^{9}, 12$ Under some situations, choice of NSAIDs is very obvious. Stroke prevention, post-myocardial infarction prophylaxis, and patient with atrial fibrillation are therapeutic situations where aspirin is the drug of choice because of its unique antiplatelet property of acetylating and causing irreversible inactivation of cyclooxygenase- 1 isoform in the platelets. ${ }^{1,24}$ Other NSAIDs inactivate this enzyme reversibly and therefore do not cause sustained antiplatelet effects. Aspirin has also been adequately studied in the chemoprevention of colon cancer. ${ }^{10,25}$ Mefenamic acid is supposed to relieve the pain of dysmenorrhoea better than other NSAIDs, although GI side effects often limit its use. ${ }^{12}$

Avoid using multiple NSAIDS and consider ulcer prophylaxis in high-risk groups: Some physicians consider combination of NSAIDs in the treatment of inflammatory joint diseases. There is little evidence to support this practice because therapeutic benefits do not add but side effects do. Moreover there is no evidence that fixed dose combinations of NSAIDs are superior to individual drugs in the long-term management of arthritis. Similarly, use of concomitant gastrotoxic drugs should be avoided, e.g., corticosteroids and NSAIDs. ${ }^{6,26}$

NSAID use in children: Choice of NSAIDs in children is generally restricted to paracetamol, aspirin, naproxan, and now nimesulide. ${ }^{12,27}$ Although nimesulide has been shown to be superior to the existing drugs in childhood febrile illnesses like upper respiratory infections, but it is more costly than the conventional NSAIDs. Aspirin is not recommended as a routine analgesic and antipyretic drug in childhood viral illness because of fear of Reyes syndrome. ${ }^{27}$ However, it enjoys its reputation as an anti-inflammatory agent in the management of rheumatic fever and childhood arthropathies.

Consider substitution: If there is no response with one drug: Surprisingly, NSAIDs have large inter-patient variations, reasons that are not entirely clear. Even when drugs are from the same chemical family or are structurally similar, they can be substituted. ${ }^{4,28}$ One patient may respond to one agent of one class but may not respond to another agent of the same class. Determination of the therapeutically effective dose for a particular patient is difficult and is often based on 'hit and trial' method. ${ }^{17}$ Treatment should be started on low dose and response should be awaited. If response is adequate, treatment is continued for one week as most side effects of NSAIDs appear in the first week. In the case of no response, change of NSAIDs should be considered. Persistent dyspepsia is one of the most frequent side effects of NSAIDs and with few exceptions it can be an indicator of onset of future gastrointestinal (GI) toxicity. ${ }^{23}$ Newer agents like celecoxib, nabumatone, and etodolac have been shown to be almost 4-fold less GI toxic than the older ones. Studies regarding the GI safety of nimesulide have not shown the reduced risk of complications.

Topical or systemic administration: Topical NSAIDs represent an attractive alternative to systemically administered drugs. Studies have shown that topically applied NSAIDs directly get to the synovial fluid, menisci, and articular cartilages and generally, $70-80 \%$ of the plasma concentration reaches the articular tissues. ${ }^{10,29}$ Interestingly, in one study, the topically applied NSAID concentrated in the menisci and cartilage to about 20-30 folds of the systemic concentration. Although the mechanisms through which they reach the joints remain to be exactly determined, the reported plasma concentration is generally less than $15 \%$ of the systemic concentration.

\section{NSAID use in developing countries}

The sales of NSAIDs include both indirect sales from wholesalers and direct sales from manufacturers. Data has been obtained from the IMS Health on the mass of individual NSAIDs sold in 2014 in 13 developing countries in the South Asian, Southeast Asian, and Asian Pacific regions (Multinational Integrated Data Analysis, MIDAS). The countries included in the analyses were: Australia, Bangladesh, China, China (Hong Kong), Indonesia, Malaysia, New Zealand, Pakistan, Philippines, Singapore, Taiwan, Thailand, and Vietnam., ${ }^{910}$ In some countries, hospital audits are based on data sourced from hospital pharmacies. In each country, the sampling data are projected to estimate sales for the whole country. The data reflected retail pharmacy and hospital sales in all countries except Bangladesh and Pakistan (retail pharmacy sales) and China (hospital sales). The defined daily doses (DDD) established by the WHO Collaborating Centre (WHOCC) for Drug Statistics Methodology, permit comparisons of use between different drugs and across different countries. ${ }^{10,18}$ It is possible to calculate the numbers of DDD of individual NSAIDs for 
each country using the values published by the WHOCC..$^{18}$ Data on NSAID prescriptions dispensed in the community in England during 2011 were obtained from public prescription cost analysis reports. ${ }^{10}$

The WHO Model List of Essential Medicines includes three drugs, paracetamol, acetyl salicylic acid (aspirin), and ibuprofen, in the category "non-opioids and non-steroidal anti-inflammatory medicines', ${ }^{9,10}$ Of the 100 countries with EMLs published on the WHO website, most included fewer than six agents in this class. The NSAIDs most commonly recommended were: aspirin (88 countries), ibuprofen ( 90 countries), diclofenac ( 74 countries), indomethacin (56 countries), and naproxen (27 countries)..$^{10}$

NSAIDs with a high risk of cardiovascular complications are widely used in developing countries. Diclofenac and etoricoxib together account for approximately one-third of all sales of NSAIDs in the 15 countries included in our analysis. ${ }^{1,29}$ There was no difference between high and low-income countries. Diclofenac was by far the most popular NSAID, despite having an RR identical to rofecoxib, ${ }^{9}$ which was withdrawn from world markets over ten years ago owing to cardiovascular toxicity. ${ }^{30,31}$ While the popularity of diclofenac in high-income countries is well known, to our knowledge this is the first report that highlights the risks associated with its dominant market position in low- and middle-income countries. Etoricoxib is the other high risk NSAID that features in this study. While there is limited information on its cardiovascular risk an updated meta-analysis, there is a doubling of cardiovascular risk compared with non-use. ${ }^{9}$ In a large head-to-head randomized clinical trial, it had an identical cardiovascular risk to diclofenac. ${ }^{22}$ Currently, etoricoxib accounted for $28 \%$ of NSAID sales in Singapore, and $14 \%$ in Bangladesh, Hong Kong, and Malaysia. The WHO Model List of Essential Medicines provides limited guidance for selection of NSAIDs on EMLs. ${ }^{15}$ It includes aspirin and ibuprofen, but offers no advice on their safety or cost-effectiveness relative to each other or to other NSAIDs. There is a gap of information on the risk profiles of patients taking NSAIDs. However, the large and consistent volumes of use of high risk NSAIDs make it very likely that these drugs are being taken by substantial numbers of individuals at high risk of serious cardiovascular events. ${ }^{9}$ NSAID recommendations on national EMLs should be based on the optimum balance of benefit and harm and give preference to low risk drugs, in particular to ibuprofen and naproxen. Diclofenac has no advantage in terms of gastrointestinal safety ${ }^{11}$ and it has a clear cardiovascular disadvantage. ${ }^{9}$ Given the availability of safer alternatives, diclofenac should be de-listed from national EMLs.

\section{Effects of NSAIDS}

\section{Analgesic and antipyretic action}

Aspirin is a weaker analgesic than morphine-type drugs, that is Aspirin 600mg<codeine 60mg < morphine 6mg. Aspirin relieves inflammatory, tissue injury related, connective tissue and integument pain but is relatively ineffective in severe visceral and ischemic pain ${ }^{9}$ The analgesic action is mainly due to peripheral pain receptors and prevention of PG mediated sensitization of nerve endings. A central subcortical action, raising threshold to pain perception also contributes. No sedation, tolerance, and dependence are produced. Aspirin resets the hypothalamic thermostat and rapidly reduces fever by promoting heat loss (sweating, cutaneous vasodilation), but does not decrease heat production. ${ }^{12}$

\section{Acid-base/ electrolyte balance}

Antiinflammatory doses produce significant changes. Initially respiratory stimulation predominates and tends to wash out $\mathrm{CO}_{2}$ despite increased production and the result is respiratory alkalosis, which is compensated by increased renal excretion of $\mathrm{HCO}_{3}$ - (with accompanying $\mathrm{Na}^{+}, \mathrm{K}^{+}$, and water). Most adults treated with 4-6g/ daily of Aspirin stay in a state of compensated respiratory alkalosis. Still higher doses cause respiratory depression with $\mathrm{CO}_{2}$ retention, while excess $\mathrm{CO}_{2}$ production continues to develop respiratory acidosis. To this is added dissociated salicylic acid as well as metabolic acid (because there is rebound depression). It develops uncompensated metabolic acidosis. Dehydration occurs in poisoning due to increased water loss in urine.

\section{Cardiovascular Risk of NSAID}

NSAIDs aside from aspirin, both newer selective COX-2 inhibitors and traditional anti-inflammatories, increase the risk of myocardial infarction and stroke They are not recommended for those who have had a previous heart attack as they increase the risk of death and recurrent MI. ${ }^{1,3}$ Evidence indicates naproxen may be the least harmful out of these Apart aside from (low-dose) aspirin are associated with a doubled risk of heart failure in people without a history of cardiac disease. ${ }^{30}$ In people with such a history, the use of NSAIDs was associated with a more than 10 -fold increase in heart failure. ${ }^{8,17}$ If this link is proven causal, researchers estimate that NSAIDs would be responsible for up to 20 percent of hospital admissions for congestive heart failure. In people with heart failure, NSAIDs increase mortality risk (hazard ratio) by approximately 1.2-1.3 for naproxen and ibuprofen, 1.7 for rofecoxib and celecoxib, and 2.1 for diclofenac.,

\section{Respiratory action}

At antiinflammatory doses respiration is stimulated by peripheral (increased $\mathrm{CO}_{2}$ production) and central (increased sensitivity of respiratory centre to $\mathrm{CO}_{2}$ ) action. Hyperventilation is prominent in salicylate poisoning. Further raise in the salicylate level causes respiratory depression and failure, and death.

\section{Gastro-intestinal tract effect}

Aspirin and its metabolite salicylic acid irritate gastric mucosa and cause epigastralgia, nausea, and vomiting. In higher doses it also stimulates CTZ. Aspirin (pKa 3.5) remains unionized and diffusible in the acid gastric juice, but on entering the mucosal cell ( $\mathrm{pH} 7.1)$ it ionizes and becomes indiffusible. This "ion trapping" in the gastric mucosal cell enhances gastric toxicity. Further, Aspirin partial contact with gastric mucosa promotes local back diffusion of acid, respectively focal necrosis of mucosal cells and capillaries, acute ulcers, erosive gastritis, congestion, and microscopic haemorrhages. The occult blood loss in stools is increased with any dose of Aspirin, averaging $5 \mathrm{ml} / 24 \mathrm{~h}$ at anti inflammatory doses. .Soluble Aspirin tablets containing calcium carbonat+citric acid and other buffered preparations have less gastric toxicity Use of NSAIDs increases risk of having a range of gastrointestinal (GI) problems ${ }^{13}$ When NSAIDs are used for pain management after surgery they cause increased risk of kidney problems. The mechanism by which NSAIDs may induce mucosal injury has been illustrated in Figure 1. The mechanism involves endothelial effects, epithelial effects, direct toxicity ion trapping, ulcer and erosion..$^{13}$

\section{Anti-inflammatory action}

This action is exerted at high daily doses of Aspirin (3 to $6 \mathrm{~g}$ ). Clinical symptoms of inflammation are suppressed, but prolongation of the under-lying disease in rheumatoid arthritis, rheumatic fever, and osteoarthritis is not affect. 


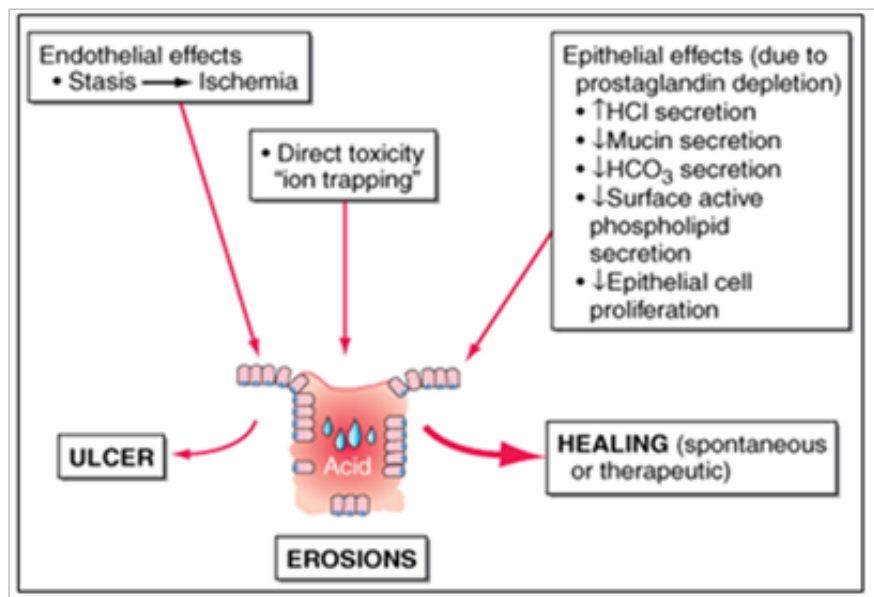

Figure I Mechanism by which NSAIDs may induce mucosal injury.

Metabolic effects: The action of Aspirin and other NSAIDs are significant only at anti-inflammatory doses. The cellular metabolism is increased, particularly at the skeletal muscles, due to the uncoupling of oxidative phosphorylation resulting from the increase in heat production. There is increased utilization of glucose and blood sugar may decrease particularly in diabetics and liver glycogen is depleted. However, hyperglycemia is often seen at toxic doses: this is due to central sympathetic stimulation and release of adrenaline and GCS. Chronic use of large doses cause negative nitrogen balance by increased conversion of protein to carbohydrate Plasma free fatty and cholesterol are reduced.

Urea excretion effect: The administration of Aspirin in high doses may reduce renal tubular excretion of urate can are trans-ported by the same mechanism. ${ }^{5}$ The widespread use of NSAIDs has shown the adverse effect of the drug becoming increasingly common. An estimated $10-20 \%$ of NSAID patients experience dyspepsia and in the 1990s high doses of prescription of NSAIDs were associated with serious upper gastrointestinal adverse events, including bleeding. There are issues of debate over the benefits and risks of the use of NSAIDs for the treatment of chronic musculoskeletal pain.,30 Each drug has a benefit-risk profile and balancing the risk of no treatment with the competing potential risks of various therapies is the responsibility of the clinician. In the last decade most deaths associated with gastric bleeding have declined. NSAIDs like most drugs, has the potential to interact with other medications. The case of concurrent use of NSAIDs and the antibiotics such as quinolone may increase the risk of quinolones' adverse central nervous system effects, including seizure.

Cardiovascular system effect: Very high doses of Aspirin have been shown to increase cardiac output to meet increased peripheral oxygen demand and can cause direct vasodilatation. ${ }^{1,5}$ Toxic doses suppress the vaso-motor centre and lowered blood pressure due to increased cardiac work as well as sodium and water retention. ${ }^{5}$

Other combinational risk: If a COX-2 inhibitor is taken, it is not advisable to take traditional NSAID, that is the over the counter prescription at the same time. ${ }^{31}$ Also, subjects on daily aspirin therapy must be careful if they also use other NSAIDs, as these may inhibit the cardioprotective effects of aspirin. Rofecoxib (Vioxx) has been shown to produce less gastrointestinal adverse drug reactions (ADRs) when compared with naproxen. ${ }^{31}$ The VIGOR trial as the case may be, raised the issue of the cardiovascular safety of the coxibs. A statistically significant increase in the incidence of myocardial infarction was observed in subjects on rofecoxib. ${ }^{5}$

Sexual dysfunction risk: Clinical trials conducted on Finish subjects in 2005 lined long term use of over three months of NSAIDs with an increased risk of erectile dysfunction. ${ }^{24}$ From the study males who used NSAIDs on a regular basis were more at risk of erectile dysfunction. The relationship NSAID use and erectile dysfunction still existed after controlling for several conditions. However, the study was observational and not controlled, with low original participation rate, potential participation bias, and other uncontrolled factors. The authors warned against drawing any conclusion regarding cause. ${ }^{24}$

\section{Inflammatory Bowel Disease (IBD)}

NSAIDs should be used with caution in individuals with inflammatory bowel disease such as Crohn's disease, due to their possibilities to cause gastric bleeding and form ulceration in the gastric lining. Pain reliever such as paracetamol or drugs containing that contains that slows down bowel movements are safer drugs for pain relief in IBD. ${ }^{13,32}$ NSAIDs are also associated with a fairly high incidence of renal adverse drug reactions. The mechanism of these renal ADRs is due to changes in renal haemodynamics (kidney blood flow), ordinarily mediated by prostaglandins, which are affected by NSAIDs. Prostaglandins normally cause vasodilation of the afferent arterioles of glomeruli. This helps maintain normal glomerular perfusion and glomerulus filtration rate, an indicator of renal functions. This is usually important in renal failure where the kidney is trying to maintain renal perfusion pressure by elevated angiotensin II levels. ${ }^{13}$ At these elevated levels, angiotensin II also constricts the afferent arteriole into the glomerulus in addition to the efferent arteriole it normally constricts. Prostaglandins serve to dilate the afferent arteriole; by blocking this prostaglandin-mediated effect, particularly in renal failure, NSAIDs cause unopposed constriction of the afferent arteriole and decreased RPF (renal perfusion pressure). These agents may also cause renal impairment, especially in combination with other nephrotoxic agents. Renal failure is especially a risk if the patient is also concomitantly taking an ACE inhibitor which removes angiotensin II's vasoconstriction of the efferent arteriole and a diuretic which drops plasma volume, and thereby RPF), the so-called "triple whammy" effect. ${ }^{32,33}$

\section{Photosensitivity}

This a commonly overlooked adverse effect of many of the NSAIDs The 2-arylpropionic acids are the most likely to produce photosensitivity reactions, but other NSAIDs have also been implicated including piroxican, diclofenac and benzydamine. The Benoxaprofen drug since withdrawn from the market due to hepatotoxicity, has been the most photoactive NSAID identified. ${ }^{31}$ The mechanism of photosensitivity, responsible for the high photo activity of the 2-arylpropionic acids, is the ready decaboxylation of carboxylic acid moiety. The specific absorbance characteristics of the different chromophoric-2 aryl substituents, affects the decarboxylation mechanism. ${ }^{1}$

\section{NSAID in pregnancy}

NSAIDs are not recommended during pregnancy, particularly during the third trimester. While NSAIDs as a class are not direct teratogens, they may provoke premature closure of the fetal ductus 
arteriosus and renal ADRs in the fetus. NSAID are also linked to premature birth and miscarriage ${ }^{27}$ Aspirin, however, is used together with heparin in pregnant women with antiphospholipid antibodies. Indomethacin is also used in pregnancy to treat polyhydramnios by reducing fetal urine production via inhibiting fetal renal blood flow. In contrast paracetamol is regarded as being safe and well-tolerated during pregnancy, however studies have shown that there may be associated male infertility in the unborn ${ }^{27}$ The doses must be taken as prescribed, to avoid potential risk of hepatotoxicity.

\section{Drug interactions}

NSAIDs reduce renal blood flow and thereby decrease the efficacy of diuretics, and inhibit the elimination of lithium and methotrexate..$^{32}$ NSAIDs cause hypocoagulability, which may be serious when combined with other drugs that also decrease blood clotting, such as warfarin. NSAIDs may aggravate hypertension and thereby antagonize the effect of antihypertensive drugs such as the ACEinhibitors. ${ }^{18}$ NSAIDs may also interfere and reduce efficiency some antidepressants. Most wisely used NSAIDs are known to enhance endocannabinoid signaling by blocking the anandamide-degrading membrane enzyme fatty acid amide hydrolase (FAAD). ${ }^{17}$ The effect of drug interaction has been illustrated in Table 1 where for example the class of drugs lead to some particular results. For example diuretics will result in decreased diuresis and beta blocker can result to decrease antihypertensive effect. ${ }^{13}$

Table I Drug interactions with NSAIDs. ${ }^{13}$

\begin{tabular}{ll}
\hline Drugs & Result \\
\hline Diuretics & Decrease diuresis \\
Beta-blockers & Decrease antihypertensive effect \\
ACE inhibitors & Decrease antihypertensive effect \\
Anticoagulants & Increase of Gl bleeding \\
Sulfonylurea & Increase hypoglycemic risk \\
Cyclosporine & Increase nephrotoxicity \\
Alcohol & Increase of Gl bleeding \\
\hline
\end{tabular}

\section{Mechanism of action NSAIDs}

Most NSAIDs act as nonselective inhibitors of the enzyme carboxylase (COX), inhibiting both the cyclooxygenase-1 (COX1) and cyclooxygenase-2 (COX-2) isoenzymes. This inhibition is competitively reversible as opposed to the mechanism of aspirin, which is irreversible inhibition. ${ }^{10,13}$ COX catalyzes the formation of prostaglandin and thromboxane and thromboxane from arachidonic acid that is itself derived from the cellular phospholipid bilayer by phospholipase A Prostaglandins act as messenger molecules in the process of inflammation. COX-1 is a constitutively expressed enzyme with a "house-keeping" role in regulating many normal physiological processes. One of these is in the stomach lining, where prostaglandins serve a protective role, preventing the stomach mucosal from being eroded by its own acid. COX-2 is an enzyme facultative expressed in inflammation, and it is inhibition of COX-2 that produces the desirable effects of NSAIDs. The mechanism of action of NSAID is illustrated in Figure 2.

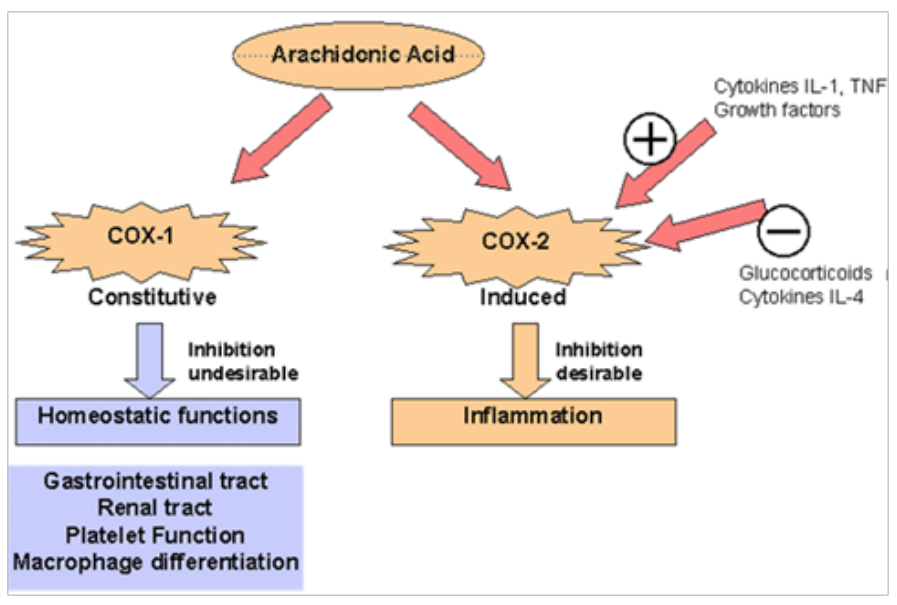

Figure 2 Mechanism of action of NSAIDs.

\section{Pharmacokinetics}

Most nonsteroidal anti-inflammatory drugs are weak acids, with a pKa of 3-5. They are absorbed well from the stomach and intestinal mucosa. They are highly protein-bound in plasma (typically $>95 \%$ ), usually to albumin, so that their volume of distribution typically approximates to plasma volume. ${ }^{30}$ Most NSAIDs are metabolized in the liver by oxidation and conjugation to inactive metabolites that typically are excreted in the urine, though some drugs are partially excreted in bile. Metabolism may be abnormal in certain disease states, and accumulation may occur even with normal dosage. Ibuprofen and diclofenac have short half-lives (2-3 hours). ${ }^{30}$ Some NSAIDs (typically oxicams) have very long half-lives (e.g. 20-60 hours).The pharmacokinetics profile of NSAIDs is illustrated in Figure 3, and as indicated for oral administration of NSAIDs most NSAIDs are weak acids and absorbs well in the stomach and intestinal mucosa. They are also metabolized in the liver by oxidation and conjugation, and $95 \%$. bound to plasma protein, with high bioavailability. ${ }^{29,30}$

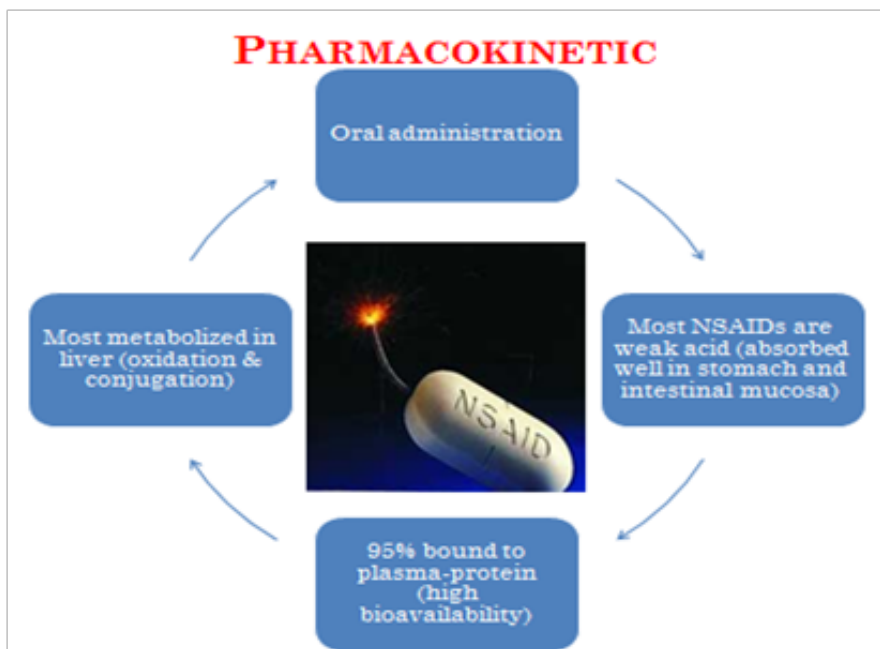

Figure 3 Pharmacological actions of non-selective NSAIDS. ${ }^{24}$ 


\section{Analgesic, antipyretic, anti-inflammatory and anti- platelet}

The clinical features of inflammation have been recognized since ancient times as swelling, redness, pain, and heat. The underlying mechanisms which produce these symptoms are complex, involving many different cells and cell products. A normal inflammatory response is essential to fight infections and is part of the repair mechanism and removal of debris following tissue damage. Inflammation can also cause disease, due to damage of healthy tissue. This may occur if the response is over-vigorous, or persists longer than is necessary. Additionally, some conditions have a previously unrecognized inflammatory component, e.g. atherosclerosis. The inflammatory response occurs in vascularzsed tissues in response to injury. It is part of the innate nonspecific immune response. Inflammatory responses require activation of leukocytes: neutrophils, eosinophils, basophils, mast cells, monocytes, and lymphocytes, although not all cell types need be involved in an inflammatory episode. The cells migrate to the area of tissue damage from the systemic circulation and become activated. ${ }^{9,10}$

\section{Diseases with a chronic inflammatory component}

Some diseases with their inflammatory cell infiltration has been describe as shown for acute respiratory distress syndrome, sarcoidosis, atherosclerosis, and many others as describe in Table 2. Most of these diseases infiltrate the neutrophils, T-cell monocytes, eosinophils or the basophils. ${ }^{15}$

Table 2 Disease with chronic inflammatory component. ${ }^{28}$

\begin{tabular}{ll}
\hline Inflammatory disease & Inflammatory cell infiltrate \\
\hline $\begin{array}{l}\text { Acute respiratory distress } \\
\text { syndrom }\end{array}$ & Neutrophil \\
Sarcoidosis & T cell, monocyte \\
Atherosclerosis & T cell, monocyte \\
Osteoarthritis & Monocyte, neutrophil \\
Inflammatory bowel disease & $\begin{array}{l}\text { Monocyte, neutrophil,T cell, } \\
\text { Eosinophil }\end{array}$ \\
Bronchial asthma & Eosinophil,T cell, monocyte, \\
basophil
\end{tabular}

\section{Some major nsaids drugs}

\section{Aspirin}

Aspirin in high dose reduces renal tubular excretion of urate (both substances are transported by the same mechanism). As analgesic (300 to $600 \mathrm{mg}$ during 6 to $8 \mathrm{~h}$ ) for head-ache, backache, pulled muscle, toothache, neuralgias as antipyretic in fever of any origin in the same doses as for analglesia. However, paracetamol and metamizole are safer, and generally preferred. Acute rheumatic fever Aspirin is the first drug of choice. Other drugs substitute Aspirin only when it fails or in severe cases. Antirheumatic doses are 75 to $100 \mathrm{mg} /$ $\mathrm{kg} / 24 \mathrm{~h}$ (resp. 4-6g daily) in the first weeks. Rheumatoid arthritis Aspirin a dose of 3 to $5 \mathrm{~g} / 24 \mathrm{~h}$ after meal is effective in most cases.
Since large doses of Aspirin are poorly tolerated for a long time, the new NSAIDs (diclofenac, ibuprofen, etc.) in depot form are preferred. Aspirin therapy in children with rheumatoid arthritis has been found to raise serum concentration trans-aminases, indicating liver damage. Most cases are asymptomatic but it is potentially dangerous An association between salicylate therapy and "Reye's syndrome", a rare form of hepatic encephalopathy seen in children, having viral infection (varicella, influenza), has been noted. Aspirin should not be given to children under 15 years unless specifically indicated, e.g. for juvenile arthritis (paracetamol is preferred). Postmyocardial infarction and poststroke patients by inhibiting platelet aggregation in low doses (100mg daily) Aspirin decreases the incidence of reinfarction. ${ }^{1,7}$ Aspirin overdose symptoms ranges from double vision, uncontrollable shaking siezures, excessive and unorganized talking fear or nervousness as indicated in Figure 4. Aspirin overdose can lead to symptoms ranging from restlessness double vision, fear and nervousness, systemic fever and others as indicated in Figure 4.

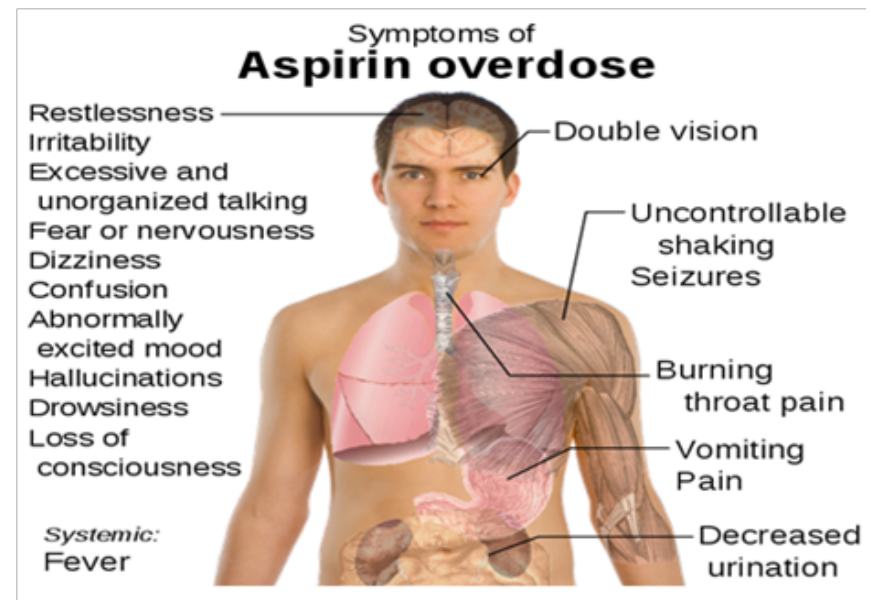

Figure 4 Symptoms of aspirin overdose.

\section{Ibuprofen}

This is a derivative of phenylpropionic acid. In doses of $2.4 \mathrm{~g}$ daily it is is equivalent to $4 \mathrm{~g}$ of Aspirin in anti-inflammatory effect. Oral ibuprofen is often prescribed in lower doses $(<2.4 \mathrm{~g} / \mathrm{d})$, at which it has analgesic but not antiinflammatory efficacy. It is available in low dose forms under several trade names (e. g. Nurofen ${ }^{\circledR}-$ film-tabl 400mg). A topical cream preparation is absorbed into fascia and muscle. A liquid gel preparation of ibuprofen provides prompt relief in postsurgical dental pain. In comparison with indometacin, ibuprofen decreases urine output less and also causes less fluid retention. It is effective in closing ductus arteriosus in preterm infants, with much the same efficacy as indomethacin. ${ }^{30}$

\section{Flurbiprofen}

Flurbiprofen is a propionic acid derivative with a possibly more complex mechanism of action than other NSAIDs. Its (S) (-) enantiomer inhibits COX nonselectively, but it has been shown in rat tissue to also affect TNF- and $N O$ synthesis Hepatic metabolism is extensive. It does demonstrate enterohepatic circulation. The efficacy of flurbiprofen at dosages of $200-400 \mathrm{mg} / \mathrm{d}$ is comparable to that of Aspirin and other NSAIDs for patients with rheumatoid arthritis, ankylosing spondylitis, gout, and osteoarthritis. Flurbiprofen i.v. is effective for perioperative analgesia in minor ear, neck, and nose surgery and in lozenge form for sore throat Its adverse effect profile is similar to other NSAIDs. 


\section{Ketoprofen}

Ketoprofen is a propionic acid derivative that inhibits both COX (nonselectively) and lipoxygenase.Concurrent administration of probenecid elevates ketoprofen levels and prolongs its plasma half-life. The effectiveness of ketoprofen at dosages of 100 $300 \mathrm{mg} / \mathrm{d}$ is equivalent to that of other NSAIDs in the treatment of rheumatoid arthritis, osteoarthritis, gout, dysmenorrhea, and other painful conditions. In spite of its dual effect on prostaglandins and leukotrienes, ketoprofen is not superior to other NSAIDs. Its major adverse effects are on the GIT and the CNS.

\section{Indometacin}

This is a potent nonselective COX inhibitor and may also inhibit phospholipase $\mathrm{A}$ and $\mathrm{C}$, reduce neutrophil migration, and decrease $\mathrm{T}$ cell and B cellproliferation. Probenecid prolongs indometacin's half-life by inhibiting both renal and biliary clearance. Indometacin is indicated for use in juvenile rheumatoid arthritis, gout and ankylosing spondylitis, postepisio-tomy pain, etc. It has been used to treat patent ductus arteriosus. An ophthalmic preparation seems to be efficacious for conjunctival inflammation and to reduce pain after traumatic corneal abrasion. Gingival inflammation is reduced after administration of indometacin oral rinse. A high incidence (up to $50 \%$ ) of GI and CNS side effects is produced: GI bleeding, diarrhoea, frontal headache, mental confusion, etc. Diclofenac is a phenylacetic acid derivative. A $0.1 \%$ ophthalmic preparation is recommended for prevention of postoperative ophthalmic inflammation and can be used after intraocular lens implantation and strabismus surgery. A topical gel containing 3\% diclofenac is effective for solar keratoses. Diclofenac in rectal suppository form can be considered a drug of choice for analgesia and postoperative nausea. It is also available for intramuscular and oral administration. Side effects occur in approximately $20 \%$ : GI distress and occult bleeding, gastric ulceration. A preparation combining diclofenac and misoprostol decreases upper GI ulceration but may result in diarrhea.

\section{Piroxicam}

This is an oxicam (enolate derivative), is a nonselective COX-1/ COX-2 inhibitor that at high concentrations also inhibits polymorphonuclear leukocyte migration, decreases oxygen radical production, and inhibits lymphocyte function. Its long half-life permits once-daily dosing. Piroxicam can be used for the usual rheumatic indications. Toxicity includes GI symptoms (20\% of patients), dizziness, and tinnitus, and headache, rash. .When piroxicam is used in dosages higher than $20 \mathrm{mg} / \mathrm{d}$, an increased incidence of peptic ulcer and bleeding is encountered. This risk is as much as 10 times higher with piroxicam than with other NSAIDs.

\section{Metamizole}

This is a derivative of pyrazolone. It is a potent and promptly acting analgesic, antipyretic, and spasmolytic but has poor antiinflammatory and not uricosuric activity. Analgin can be given orally, i.m. as well as i.v. (very slowly). Pain at the i.m. injection site and rarely abscess can occur. Occasionally an i.v. injection produces fall in BP. Few cases of agranulocytosis were reported and metamizole was banned in the USA and some European country. However, it has been extensively used in Bulgaria and much other European country, as well as in India and Russia. Adverse reaction data collected over four decades shows that the risk of serious toxicity with metamizole is very low than with Aspirin or many other NSAIDs.

\section{Paracetamol use}

Although equivalent to Aspirin as an effective analgesic and antipyretic agent, paracetamol differs in that it lacks antiinflammatory roperties. It does not affect uric acid levels and lacks plateletinhibiting properties. The drug is useful in mild to moderate pain: headache, myalgia, postpartum pain. Paracetamol alone is inadequate therapy for inflammatory conditions such as rheuma- toid arthritis, although it may be used as an analgesic adjunct to antiinflammatory therapy. For mild analge-sia, paracetamol is the preferred drug in patients' aller-gic to Aspirin or when salicylates are poorly tolerated it is preferable to Aspirin in patients with hemophilia or a history of peptic ulcer and bronchospasm. It is preferred to Aspirin in children with viral infections.

\section{Comparative action between COX inhibitors}

Studies on the comparative action between COX inhibitor has been done on some pharmacological actions and diseases response as shown in Table 3. For example the analgesis action shows a response with COX-1/COX-2 inhibitors and a stronger response with COX-2 inhibitors.

Table 3 Comparative action between COX inhibitors

\begin{tabular}{|c|c|c|}
\hline $\begin{array}{l}\text { Comparative action } \\
\text { between COX inhibitors }\end{array}$ & $\begin{array}{l}\text { COX-I/COX-2 } \\
\text { inhibitors }\end{array}$ & COX-2 inhibitors \\
\hline I.Analgesic action & $(+)$ & $(+)(+)$ \\
\hline 2.Antiinflammatory action & $(+)$ & $(+)(+)$ \\
\hline 3.Antiinflammatory action & $(+)$ & $(+)(+)$ \\
\hline 4.Antiplatelet aggregators & $(+)$ & $(-)$ \\
\hline 5. Gastric mucosal damage & $(+)(+)(+)$ & $(+)$ \\
\hline 6. Renal salt/water retention & $(+)$ & $(+)$ \\
\hline $\begin{array}{l}\text { 7.Delay/prolongation of } \\
\text { labour }\end{array}$ & $(+)(+)$ & $(+)$ \\
\hline 8. Infertility & $(-)$ & $(+)(+)$ \\
\hline 9. Ductus arteriosus closure & $(+)$ & $?$ \\
\hline 10.Aspirin-like asthma & $(+)$ & $?$ \\
\hline I I. Cardio toxicity & $(-)$ & $(+)(+)$ \\
\hline
\end{tabular}

\section{Nsaid poisoning \\ Acute paracetamol poisoning}

This occurs especially in small children who have low hepatic glucuronide conjugating ability. If a large dose $(>150 \mathrm{mg} / \mathrm{kg}$ or $>10 \mathrm{~g}$ in adult) is taken, serious toxicity can occur as shown in Figure 5 \&6. On the metabolism and toxicity of paracetabol. The letal dose is $250 \mathrm{mg} / \mathrm{kg}$. N-acetyl-p-benzoquinoneimine (NABQI) is a highly reactive arylating metabolite of paracetamol which detoxicated by conjugation with glutathione. When a very large doses of paracetamol 
are taken, the glucuroconjugation capacity is saturated, more NABQI is formed, hepatic glutathione is depleted and NABQI binds covalently to proteins in liver cells (and renal tubules) causing necrosis. In chronic alcoholics even $5-6 \mathrm{~g} / \mathrm{d}$ taken for a few days can result in hepatotoxicity because ethanol induces CYP 2E2, that metabolizes paracetamol, to NABQI. Treatment needs activated charcoal, given orally or through the tube to prevent GI absorption, and acetylcysteine $(150 \mathrm{mg} / \mathrm{g}$ by i.v. infusion).

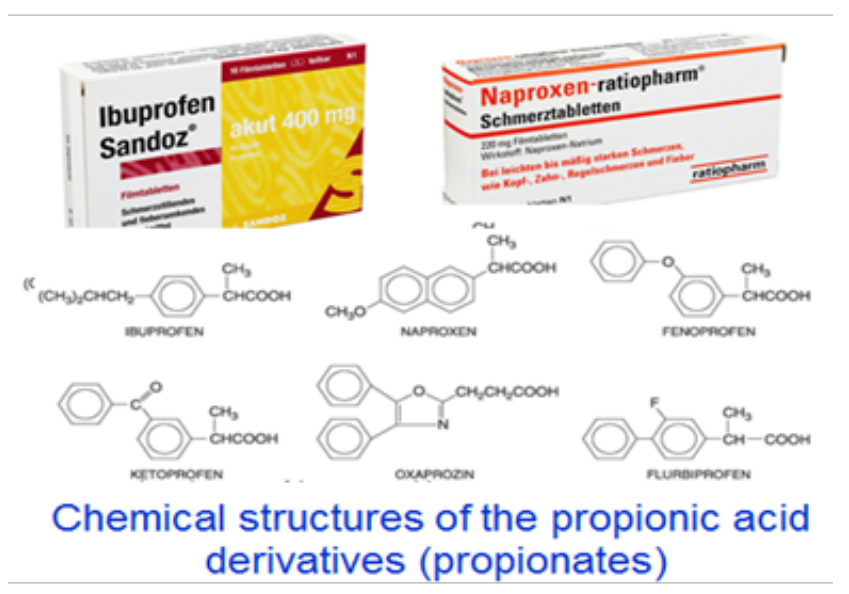

Figure $\mathbf{5}$ The chemical structures of the propionic acid derivatives (propionates)..$^{30}$

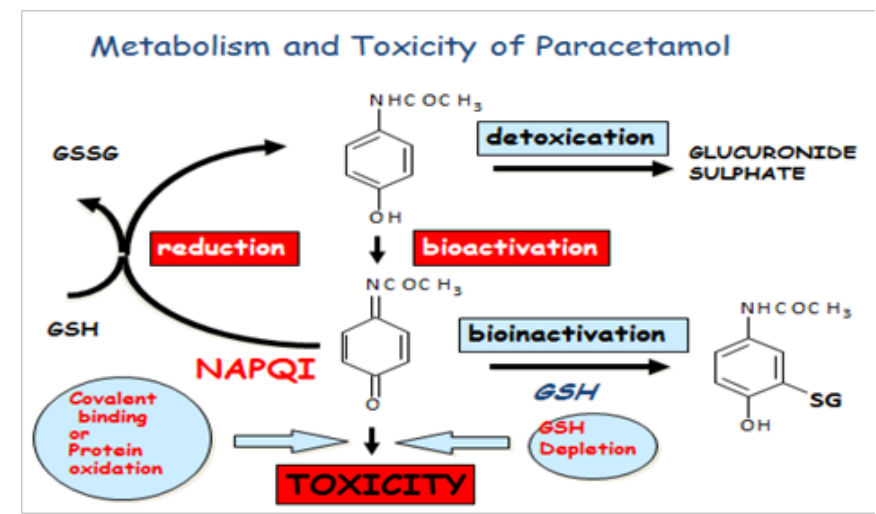

Figure 6 The metabolism and toxicity of paracetabol.

\section{Conclusion}

It is clear that the COX-2 inhibitors are safer, better tolerated, and equally efficacious, but many clinical issues need to be fully resolved. The fact that NSAIDs in developing economy has been shown to be readily available as over the counter medication predisposes the drug to heavy abuses and the side effect as in the case of paracetamol overdose leads to frequent hospitalization of victims or in some cases the effects are not noticed till disaster occurs. The non-steroidal antiinflammatory drugs (NSAIDs) belong to a drug class that groups together drugs that provide analgesic (pain-killing) and antipyretic (fever-reducing) effects. In higher doses these drugs produce antiinflammatory effects. The analgesic and anti-inflammatory properties acts by inhibiting two recognized isoenzymes of prostaglandin cycl-oxygenase (COX), which are subgroups COX 1 and COX 2.The pharmacodynamic action of these drugs is mostly base on the inhibition of COX2, while the adverse reactions are largely due to COX1 inhibition.. Currently, there are still information gaps on the risk of heart failure associated with the use of individual NSAIDs (both COX 2 inhibitors and traditional NSAIDs) in clinical practice in resource poor communities, and especially on their dose-response associations. The most prominent members of this group of drugs, are aspirin, ibuprofen, naproxen, that are all available over the counter in most countries. Paracetamol (acetaminophen) is generally not considered an NSAID because it has only little anti-inflammatory activity.

\section{Acknowledgements}

None.

\section{Conflict of interest}

The author declares no conflict of interest.

\section{References}

1. Grosser T, Fries S, FitzGerald GA. Biological basis for the cardiovascular consequences of Cox 2 inhibition: therapeutic challenges and opportunities. J Clin Invest. 2006;116(1):4-15.

2. Hernandez-Diaz S, Lorenzo V, Rodriguez GL. Non-steroidal antiinflammatory drugs and the risk of acute myocardial infarction. Basic Clin Pharmacol Toxicol. 2006;98(3):266-274.

3. McGettigan P, Henry D. Cardiovascular risk and inhibition of cyclooxygenase: a systematic review of the observational studies of selective and nonselective inhibitors of cyclo-oxygenase. JAMA. 2006;296(13):1633-1644.

4. McGettigan P, Henry D. Cardiovascular risk with non-steroidal antiinflammatory drugs: systematic review of population-based controlled observational studies. PLoS Med. 2011;8(9):e1001098.

5. Scheiman JM, Hindley CE. Strategies to optimize treatment with NSAIDs in patients at risk for gastrointestinal and cardiovascular adverse events. Clin Ther. 2010;32(4):667-677.

6. Rostom A, Muir K, Dube C, et al. Prevention of NSAID-related upper gastrointestinal toxicity: a meta-analysis of traditional NSAIDs with gastroprotection and cox-2 inhibitors. Drug Healthcare Patient Safety. 2009;1:47-71.

7. Strand V. Are COX-2 inhibitors preferable to non-selective nonsteroidal anti-inflammatory drugs in patients with risk of cardiovascular events taking low dose aspirin? Lancet. 2007;370(9605):2138-2151.

8. Garcia Rodriguez L, Tacconelli S, Patrignani P. Role of dose potency in the prediction of risk of myocardial infarction associated with nonsteroidal anti-inflammatory drugs in the general population. $\mathrm{J} \mathrm{Am} \mathrm{Coll}$ Cardiol. 2008;52(20):1628-1636.

9. http://whqlibdoc.who.int/hq/2011/a95053_eng.pdf

10. Essential medicines selection. Geneva: World Health Organization; 2012.

11. Fosbol EL, Folke F, Jacobsen S, et al. Cause specific cardiovascular risk associated with nonsteroidal anti-inflammatory drugs among healthy individuals. Circ Cardiovasc Qual Outcomes. 2010;3(4):395-405.

12. Deeks JJ, Smith LA, Bradley MD. Efficacy, tolerability, and upper gastrointestinal safety of celecoxib for the treatment of osteoarthritis and rheumatoid arthritis: a systematic review of randomised controlled trials. BMJ. 2002;325(7365):619-623.

13. Buttgereti F, Burwester R, Simon LS. Gastrointestinal toxic side effects of non-steroidal anti-inflammatory drugs and cyclooxygenase inhibition. Am J Med. 2001;110(3A):13S-19S.

14. Walton A. Renal aspects of treatment with conventional nonsteroidal anti-inflammatory drugs versus cyclooxygenase-2-specific inhibitors. Am J Med. 2001;110(3A):33S-42S. 
15. Emery P. Cyclooxygensase-2:A major therapeutic advance? Am J Med 2001;110(1A):42S-45S

16. Schnitzer TJ. Cycloxygense-2-specific-inhibitors: Are they safe? Am J Med. 2001;110(1A):46S-49S.

17. BuerJK. Origins and impact of the term 'NSAID. Inflammopharmacology. 2014;22(5):263-267.

18. Warden SJ. Prophylactic Use of NSAIDs by Athletes: A Risk/Benefit Assessment. Phys Sportsmed. 2010;38(1):132-138.

19. Hinz B, Cheremina O, Brune K. Acetaminophen (paracetamol) is a selective cyclooxygenase-2 inhibitor in man. FASEB J. 2008;22(2):383390.

20. Pattanittum P, Turner T, Green S, et al. Non-steroidal anti-inflammatory drugs (NSAIDs) for treating lateral elbow pain in adults. Cochrane Database Syst Rev. 2013;31(5):CD003686.

21. Lim BX, Lim CH, Lim DK, et al. Prophylactic non-steroidal antiinflammatory drugs for the prevention of macular oedema after cataract surgery. Cochrane Database Syst Rev. 2016;11:CD006683.

22. Danelich IM, Wright SS, Lose JM, et al. Safety of nonsteroidal anti inflammatory drugs in patients with cardiovascular disease". Pharmacotherapy. 2015;35(5):520-535.

23. Bombardier C, Laine L, Reicin A, et al. Comparison of upper gastrointestinal toxicity of rofecoxib and naproxen in patients with rheumatoid arthritis. New England Journal of Medicine. 2000;343(21):1520-1528

24. Shiri R, Koskimäki J, Häkkinen J, et al. Effect of Nonsteroidal AntiInflammatory Drug Use on the Incidence of Erectile Dysfunction. Journal of Urology. 2006;175(5):1812-1816.
25. Gleason JM, Slezak JM, Jung $H$, et al. Regular Nonsteroidal Anti-Inflammatory Drug Use and Erectile Dysfunction. J Urol. 2011;185(4):1388-1393.

26. Higuchi K, Umegaki E, Watanabe T, et al. Present status and strategy of NSAIDs-induced small bowel injury. Journal of Gastroenterology. 2009;44(9):879-888.

27. Nakhai-Pour HR, Broy P, Sheehy O, et al. Use of non aspirin nonsteroidal anti-inflammatory drugs during pregnancy and the risk of spontaneous abortion. CMAJ. 2011;183(15):1713-1720.

28. Kowalski ML, Makowska JS. Seven steps to the diagnosis of NSAIDs hypersensitivity: how to apply a new classification in real practice? Allergy Asthma Immunol Res. 2015;7(4):312-320.

29. Auriel E, Regev K, Korczyn AD. Nonsteroidal anti-inflammatory drugs exposure and the central nervous system. Handb Clin Neurol. 2014;119:577-584.

30. Woessner KM, Castells M. NSAID single-drug-induced reactions". Immunol Allergy Clin North Am. 2013;33(2):237-249.

31. Rainsford KD. Ibuprofen: pharmacology, efficacy and safety". Inflammopharmacology. 2009;17(6):275-342.

32. Fung M, Thornton A, Mybeck K, et al. Evaluation of the Characteristics of Safety Withdrawal of Prescription Drugs from Worldwide Pharmaceutical Markets-1960 to 1999. Therapeutic Innovation \& Regulatory Science. 2001;35(1):293-317.

33. Viljoen A, Mncwangi N, Vermaak I. Anti-inflammatory iridoids of botanical origin. Curr Med Chem. 2012;19(14):2104-2127. 